\title{
Exploring the complexity of intellectual disability in fetal alcohol spectrum disorders
}

\section{Aniruddho Chokroborty-Hoque ${ }^{\dagger}$, Bonnie Alberry $^{\dagger}$ and Shiva M. Singh*}

Molecular Genetics Unit, Department of Biology, University of Western Ontario, London, ON, Canada

Edited by:

Andrea De Giorgio, Catholic

University, Italy

\section{Reviewed by:}

Gregg Stanwood, Vanderbilt

University, USA

Alberto Granato, Catholic University, Italy

\section{*Correspondence:}

Shiva M. Singh, Molecular Genetics

Unit, Department of Biology,

University of Western Ontario, 1151

Richmond Street, London, ON

N6A5B7, Canada

e-mail: ssingh@uwo.ca

${ }^{+}$Contributed equally and are joint first authors of this manuscript.
Brain development in mammals is long lasting. It begins early during embryonic growth and is finalized in early adulthood. This progression represents a delicate choreography of molecular, cellular, and physiological processes initiated and directed by the fetal genotype in close interaction with environment. Not surprisingly, most aberrations in brain functioning including intellectual disability (ID) are attributed to either gene(s), or environment or the interaction of the two. The ensuing complexity has made the assessment of this choreography, ever challenging. A model to assess this complexity has used a mouse model (C57BL/6J or B6) that is subjected to prenatal alcohol exposure. The resulting pups show learning and memory deficits similar to patients with fetal alcohol spectrum disorder (FASD), which is associated with life-long changes in gene expression. Interestingly, this change in gene expression underlies epigenetic processes including DNA methylation and miRNAs. This paradigm is applicable to ethanol exposure at different developmental times (binge at trimesters 1, 2, and 3 as well as continuous preference drinking (70\%) of $10 \%$ alcohol by B6 females during pregnancy). The exposure leads to life-long changes in neural epigenetic marks, gene expression, and a variety of defects in neurodevelopment and CNS function. We argue that this cascade may be reversed postnatally via drugs, chemicals, and environment including maternal care. Such conclusions are supported by two sets of results. First, antipsychotic drugs that are used to treat ID including psychosis function via changes in DNA methylation, a major epigenetic mark. Second, post-natal environment may improve (with enriched environments) or worsen (with negative and maternal separation stress) the cognitive ability of pups that were prenatally exposed to ethanol as well as their matched controls. In this review, we will discuss operational epigenetic mechanisms involved in the development of intellectual ability/disability in response to alcohol during prenatal or post-natal development. In doing so, we will explore the potential of epigenetic manipulation in the treatment of FASD and related disorders implicated in ID.

Keywords: neurodevelopment, fetal alcohol spectrum disorders, mouse models, epigenetics, stress, environmental enrichment, intellectual disability, gene expression

\section{INTELLECTUAL DISABILITY}

Mental retardation is a highly diverse group of cognitive disorders. The fifth edition of the Diagnostic and Statistical Manual of Mental Disorders (5th ed.; DSM-5) characterizes mental retardation [revised as Intellectual Disability (ID) in the fifth edition) by impairments of general mental abilities that fall under the conceptual, social, and practical domains of adaptive functioning. Individuals with ID have intelligence quotient (IQ) scores below 70 , approximately two standard deviations or more below the population average score (1). They represent $1-3 \%$ of the population in Canada, and males are affected more often than females (2). Roughly two-thirds of ID individuals have mild-to-moderate impairments while the remaining third are severely affected (2). In 2006, the Participation and Activity Limitation Survey by Statistics Canada, found the largest proportion of ID occurs in the 15-24 age group $(29.3 \%)(2)$. The survey also found that compared to individuals with physical disabilities $(52.7 \%)$, people with intellectual disabilities are far less likely to be employed (26.1\%) with an inverse trend of socio-economic status with respect to prevalence (2). In America, the lifetime costs are expected to be near $\$ 50$ billion for individuals born in 2000 with ID (3).

Intellectual disability and its variable manifestations are often attributed to aberrations in neurodevelopment that are complex, poorly understood, and long lasting in mammals. It begins early during embryonic development but may take years to complete and is finalized in early adulthood. Further, it involves a delicate choreography of cellular, molecular, and physiological processes directed by the fetal genotype in close interaction with the environment at every step, over time. Consequently, it covers periods before birth, during birth, and/or the childhood years. The causes of ID are complex and multifactorial. In some rare cases, the primary determinants of ID are known. For example, rare chromosome number defects [Down syndrome $(4,5)$ ], inherited chromosomal disorders [Fragile X syndrome (6)], and a number of single gene mutations (7-9) are known to cause a spectrum of intellectual. Unfortunately, ID tends to be heterogeneous with 
a wide spectrum of manifestations. Additionally, neurodevelopmental exposure to a variety of drugs and chemicals can result in ID, such as cocaine, alcohol, and lead, among others (10). ID is not a single disorder, rather the result of a plethora of causations involving both genes and environment. The understanding of the developmental processes associated with ID and related abnormalities calls for a research focus on specific diagnosis potentially caused by a single known factor, an experimental model that is easier to manipulate and interpret. In this discussion, we will use fetal alcohol spectrum disorders (FASD) as a case study of the complexity of ID.

\section{FETAL ALCOHOL SPECTRUM DISORDERS}

Fetal alcohol spectrum disorder with all its manifestations results from a single initial cause, prenatal alcohol exposure (PAE). It includes mild behavioral and learning impairments, to the most severe form called Fetal Alcohol Syndrome (FAS). FAS may include ID as well as birth defects (11). The intellectual deficits in FAS and FASD are highly variable and heterogeneous. These symptoms are considered chronic, often co-occurring with other mental impairments, and manifesting during the developmental period. FASD represents one of the most common causes of learning disabilities, cognitive deficits, and ID (12). The severity of impairments is evaluated using both clinical assessments and standardized testing of intelligence.

Obtaining a diagnosis of FASD requires input from various medical professionals, with estimated costs associated with the diagnostic procedure up to $\$ 7.3$ million per year in Canada (13). Unfortunately, despite increasing public education on the dangers of PAE, the occurrence of alcohol exposed pregnancies remains a significant societal problem. In Canada, $74.4 \%$ of women surveys reported alcohol use in the past year (14). Most disappointing is the prevalence of PAE in certain high-risk groups. In Fort McMurray, Alberta, almost $50 \%$ of pregnant women surveyed reported consuming an alcoholic beverage since learning of their pregnancy (15). In a survey of women in Arctic Quebec, over 60\% of women reported alcohol consumption during pregnancy (16). While the rates of PAE are often considered high, not every reported incident of alcohol exposure results in FASD. In Canada, the more severe FAS is estimated to occur at rates of 1-2 per 1000 live births (17), while the more mild FASD occurs much more often, at a rate of 9 per 1000 (18). In northeastern Manitoba, estimates of FASD incidence are as high as 14.8 per 1000 births (19). Children entering child care systems, such as foster care and orphanages, also represent a subpopulation with higher incidence of FASD, with estimates at 60 per 1000 children (20). A 2010 study found that $48 \%$ of pregnancies in the United States were unintended (21), and over $30 \%$ of women reported consuming alcohol while pregnant (22). The Centre for Disease Control and Prevention reports FAS rates in the USA ranging from 0.2 to 1.5 per 1000 live births solidifying the position of FAS as one of the leading preventable causes of intellectual disabilities (23).

How neurodevelopmental alcohol exposure may cause ID is poorly understood. It is a critical area of research. Such studies are not always feasible in humans. In this review, we will present arguments to suggest that studies on the mechanisms in the development of intellectual disabilities could be modeled in suitable animal models using PAE. It allows coverage of prenatal as well as post-natal development. Specifically, we will focus on behavioral data to show that B6 mice offer an opportunity to assess the effect of neurodevelopmental time specific PAE on molecular processes that are affected by alcohol and may lead to the manifestation of ID and related abnormalities. Additionally, it allows controlled post-natal manipulation (negative stress or positive enrichment) on the manifestation of mental deficits in pups generated with and without PAE.

\section{MOUSE MODEL OF FASD RESEARCH}

It is understandable that most research on the mechanisms involved in the development of FASD has concentrated on animal models, particularly mice (24-27). To this end, our laboratory has established two forms of neurodevelopmental in vivo alcohol treatment in B6 mice. The first uses injections at any time during neurodevelopment on time-mated females and the second uses free choice of $10 \%$ alcohol or water as the source of liquid for pregnant females. The pregnant B6 mothers prefer $(\sim 70 \%)$ to drink a $10 \%$ ethanol in water solution over water. The two methods equate to the two forms of PAE in humans; binge (injection) at any time during pregnancy and continuous maternal drinking (preference) during pregnancy. The resultant pups from the two treatments show alcohol specific phenotypes; developmental delays, increased anxiety, learning deficits, and pronounced deficits in visuo-spatial memory (27-29). They also exhibit delayed neural reflexes, aberrant limbic coordination, elevated levels of anxiety, and spatial-memory deficits (27). To better ascertain the effects of ethanol on critical neurodevelopmental time points, we have mimicked binge-like drinking episodes at critical times, representing equivalents to the three trimesters in humans. The trimester three equivalent represents a "brain growth spurt" - dominated by synaptogenesis during the first 2 weeks in B6 newborn pups $(29,30)$. It is a period marked by the formation of extensive neural connections that form the basis for much of the cell-to-cell communication in the brain. The ability of ethanol to trigger widespread neurodegeneration during synaptogenesis is accompanied by the upregulation of stress-related and apoptosis-related genes and a down-regulation of genes related to protein synthesis, mitosis, synaptic formation, and maintenance $(28,30,31)$. The third trimester equivalent ethanol exposure also results in increased anxiety-like behavioral traits and pronounced recognition memory and visuo-spatial memory defects (29). The results show that most PAE treatments in B6 mice cause developmental as well as behavioral deficiencies that are compatible with manifestations of FASD. Additional studies regarding timing of ethanol exposure have found exposure during the first trimester equivalent leads to decreases in cerebellar volume, while second trimester equivalent exposure leads to decreased hippocampal volume (32). The model also allows further studies on specific brain regions that may offer novel insights. The hippocampus is one of the brain regions that may be important in the understanding of the complexity of FASD phenotypes. The primary role of the hippocampus is memory consolidation (33), emphasized by hippocampal lesions leading to impaired spatial learning in mice (34). PAE leads to learning and memory deficits via changes in the hippocampus (35). Ultimately, some of the behavioral effects of PAE may be a result of molecular 
changes in the hippocampus. The molecular effects of PAE have been well characterized using animal models under a plethora of conditions (various neurodevelopmental stages and different dosages of alcohol) and all of them have shown that PAE affects epigenetic and genetic processes and various neurodevelopmental pathways (36-38). A single (or in most cases, multiple) instance of alcohol exposure during fetal development can result in a lifetime of behavioral and cognitive deficits. Such results show that PAE treatments in B6 mice cause deficiencies that are comparable to the manifestations of FASD in humans.

\section{MOLECULAR ETIOLOGY OF FASD: GENE EXPRESSION AND EPIGENETIC MARISS}

The development of genomic technologies has allowed the search for molecular mechanisms underlying deficits following PAE. In both cultured neurons and in vivo evidence, ethanol has been shown to induce programed cell death 4 (PDCD4) protein synthesis, ultimately resulting in neuronal growth abnormalities in a rat model of PAE (39). Ethanol has also been shown to induce apoptosis via ceramide pathways, alongside stress-related kinases during development in cultured rat astrocytes (40). Direct treatment of ethanol on cultured neural stem cells often results in a host of changes at the level of gene expression. It includes Dnmt1, Uhrfl, Ehmt1, Ash 2 l, Wdr5, and Kdm $1 b$ transcripts that have been shown to have significantly different levels of gene expression following ethanol exposure in vitro (41).

We have attempted such studies on B6 in vivo (42). The results show that multiple ethanol-treatment paradigms that result in FASD phenotypes also show changes in gene expression $(28,30)$. Such changes occur with respect to neurodevelopmental timing of exposure. More important they are representative of genomic alterations that are dependent on the biological processes occurring at the time of ethanol exposure $(30,42)$. Interestingly, ethanol exposure initiates alterations in a set of genes (short-term effect) that primarily affect cellular compromise and apoptosis representative of ethanol's toxic effects. In the long term, however, genes affected following PAE are very different and involve various cellular functions including epigenetic processes such as DNA methylation, histone modifications, and non-coding RNA regulation that may underlie long-term changes to gene expression patterns (43). These may be initiated by ethanol-induced alterations to DNA and histone methylation, particularly in imprinted regions of the genome, affecting transcription, which is further fine-tuned by altered microRNA (44). These processes are likely complex, genome-wide, and interrelated. The epigenetic changes may be responsible for the FASD-related alterations in gene expression. Additionally, the epigenetic changes acquired may remain stable for life and maintain the manifestation of FASD.

At least two features of this system are encouraging and offer hope for people affected with FASD and related disorders. First, human brain development is not complete at birth, rather it continues for decades. More important, the neurodevelopment during this period is rather malleable and responsive to postnatal environment. Consequently, it may provide an opportunity to direct/maneuver post-natal brain development and alter the course of development of FASD and related endophenotypes. Second, the underlying epigenetic changes brought about by PAE represent an adjustable process. Specifically, DNA methylation is known to be reversible, and may be altered using different strategies. This promise and hope offered by the two features (continuity of brain development after birth and potential to change PAE epigenetic marks) have remained poorly explored in FASD-related studies. We will present preliminary results to argue that the continuum of post-natal neurodevelopment offers an opportunity to ameliorate the effect of prenatal alcohol and adjust/restore the final outcome.

\section{POST-NATAL ENVIRONMENT NEURODEVELOPMENT AND FUNCTIONING}

Mammalian neurodevelopment is a long-lasting continuum. It begins early and finalized in early adulthood. It is also closely orchestrated and sensitive to prenatal as well as post-natal environment, particularly stresses. It makes it nimble with potential to incorporate desirable post-natal experiences. The mechanism behind this potential although recognized is not fully understood. What is known is that post-natal processes contribute to the lifelong changes in behaviors and mental abilities. Also, it may result via responsiveness of the hypothalamic-pituitary-adrenal (HPA) axis (45), the primary physiological regulator of the environmental stress in mammals. Perhaps, the strongest evidence for this effect comes from post-natal handling of rodents. It involves daily separation of pups from the mother (3-15 min), a stressful event, for the first few weeks of life. Such pups show decreased stress reactivity in adulthood (46-48). Also, pups exposed to extended positive maternal care show decreased fearfulness and more modest HPA responses to stress $(49,50)$. Similar results have also been reported in non-human primates (51), and humans (52, 53). In each case, variations in post-natal conditions promote hippocampal synaptogenesis and spatial learning and memory through systems known to mediate experience-dependent neural development (54). The question of how post-natal environment causes such a dramatic effect in mammals has formed a fruitful area of research in recent years. It argues that this effect may be realized via the effectiveness of HPA axis.

An underlying mechanism behind the effect of post-natal environment is provided by studies by Michael Meaney and his collaborators. They found that increased licking and nursing by rat mothers altered DNA methylation of hippocampal glucocorticoid receptor in the pups. Further, the altered methylation is directly related to the development of HPA responses to stresses through tissue specific effects on gene expression (55). The results also emphasize that there is a critical period for such effects to be realized. It is particularly effective in early post-natal periods. This relationship between maternal care and gene expression via DNA methylation argues for environmental reprograming that is stable and may form the basis for the developmental origin of vulnerability to defects (56). These results have now been replicated in a number of mammals including humans. They argue that early life events can alter the methylation (epigenetic) state of relevant genomic regions, the expression of which may contribute to individual differences in the risk for pathology and diseases of fetal origin (57). Given this understanding, it is natural to consider post-natal enrichment in correction of any epigenetic pathology. It is particularly relevant in cases of FASD that are caused by 
alcohol-induced alterations in DNA methylation. In fact, one may postulate potential involvement of DNA methylation (58) at every step in neurodevelopment including responses to environment prenatally as well as postnatally.

The results available have allowed us to propose alterations in the sequential continuum of neurodevelopment in FASD over a longer time frame - from fertilization to maturity (Figure 1). It shows the continuum of neural development with and without prenatal alcohol that result in metabolomic changes leading to either FASD or not. It recognizes that the manifestation of this outcome is not fixed. It must follow additional development and refinement in a given post-natal environment. Once again, postnatal environment may affect the developing brain via epigenetic and metabolomic alterations. We argue that such alterations will vary and depend on the nature (heavily enriched to heavily stressful) of the post-natal environment. Consequently, the effect of post-natal environment may permit recovery from prenatal effects [enriched environments (EE)] or add additional defects (stressful environment). The model covers molecular processes that underlie the initiation, progression, and completion of neurodevelopment and any role prenatal or post-natal stress may have during gestation, birth, and post-natal development. The model recognizes that the effect of post-natal environment is not restricted to cases with PAE. Rather, it is expected to have an impact on cases where there is no exposure to alcohol. Further, although the nature of prenatal stress is well defined, the nature of post-natal environment that will have a positive and negative effect remains rather generic and needs to be carefully investigated. We will discuss this model further using ongoing experiments.

\section{ASSESSING THE POST-NATAL ENVIRONMENT ON FASD}

Fetuses exposed to alcohol prenatally have poor growth in the womb. Consequently, they are born with low birth weight (59). Further, almost none of such babies have normal brain development. They also show decreased muscle tone, poor coordination, and slow growth rate (59). Naturally, newborns with FASD are dependent on post-natal care by the mother for their future development. Also, they are often born in suboptimal families and raised in suboptimal conditions, particularly in the previously outlined high-risk subpopulations. Consequently, an unfavorable post-natal environment often provides a continuation of prenatal developmental insults thereby increasing the risk and severity of the PAE outcome. It has been argued that an enriched postnatal environment may have an ameliorating effect on the brain development in the FASD babies but the effect of post-natal environment on the development of FASD phenotypes has not been adequately explored. We will assess the results of stressful and enriched post-natal environment on the growth, development, and mental ability of newborns with diagnosed with FASD.

\section{STRESSFUL POST-NATAL ENVIRONMENT IN FASD}

Results available in the literature argue that the combined effect of PAE and post-natal stress worsen the behavioral and structural effects of alcohol exposure alone $(60,61)$. Further accumulative stressors over time may contribute to increased risk of depression in FASD via HPA axis dysregulation (62). In humans, there are sex-differences in stress regulation, in that females show greater changes in heart rate, while males exhibit more alterations in cortisol levels (63). In primates, the combination of PAE and maternal stress led to a reduction in birth weight in males, but not females - further highlighting the sex-specificity. Additionally, both sexes show HPA axis responses following maternal separation stress (64). In rodent models of PAE, the basal levels of corticosterone and adrenocorticotropin (stress-related molecules) are unaltered, but subjects are hyper-responsive to stressors in adulthood (65). Chronic stress leads to increases in corticosterone in ethanol exposed females following an acute stress event (66), and prolonged exposure to stressors in ethanol exposed males leads to overactive HPA response (65). In B6 mice, maternal separation stress on normal pups is often used to model chronic early life stress. It uses $3 \mathrm{~h}$ of separation per day from post-natal days $2-$ 14 that can result in anxiety-like behaviors in adult mice (67). The resulting mice display increased anxiety-like behaviors on open-field testing (68) similar to those observed in PAE models without maternal separation. Interestingly, behavioral abnormalities including learning and memory deficits induced by PAE and prenatal stress may be moderated by administration of BDNF (69). Further, such effects may be due to changes in hippocampal gene expression $(70,71)$. The general conclusion is that stressful postnatal environment may add deterioration on young's exposed to prenatal alcohols. The specific interaction between prenatal alcohol and stressful post-natal environment however, has not been sufficiently examined.

\section{POST-NATAL ENVIRONMENTAL ENRICHMENT IN FASD}

Prenatal alcohol causes FASD. Also, how prenatal alcohol may manifest the development of FASD is becoming apparent. One of the next logical questions in FASD research deals with the role of post-natal environment. Most FASD children are born into an environment of malnutrition and drug and nicotine abuse $(72,73)$. In addition, poor socio-economic lifestyles along-with neglectful parenting, exacerbate the behavioral and cognitive abnormalities so characteristic of FASD children. It has been hypothesized that an enriched post-natal environment may lessen the severity of the manifestation in a newborn diagnosed with FASD. An enriched post-natal environment may involve intensive physical, cognitive, and behaviorally challenging environments $(74,75)$. The repeated exposure to counseling sessions and specialized classes with an aim to develop verbal, math, and social skills helps ameliorate some, if not all behavioral and cognitive deficits. While some interventions manage to lessen stress and anxiety levels in FASD children, cognitive disabilities still remain at large. However, such rehabilitative therapies have been unsuccessful in improving the spectrum of ID in FASD. What is needed is a better understanding of the molecular events that follow rehabilitative therapies in humans. To this end, it will be desirable to answer the question: Do rehabilitative therapies target the very same affected molecular pathways that cause FASD or do they have different molecular mechanisms? Such questions are better explored using animal models.

What constitutes "rehabilitative therapies" in rodent models of FASD? Ethanol exposed rats and mice that are subjected to physically and cognitively challenging environments (EE) tend to be less stressed and have improved memory performance (76). Given how fetal alcohol exposure affects neurodevelopment, it is possible 


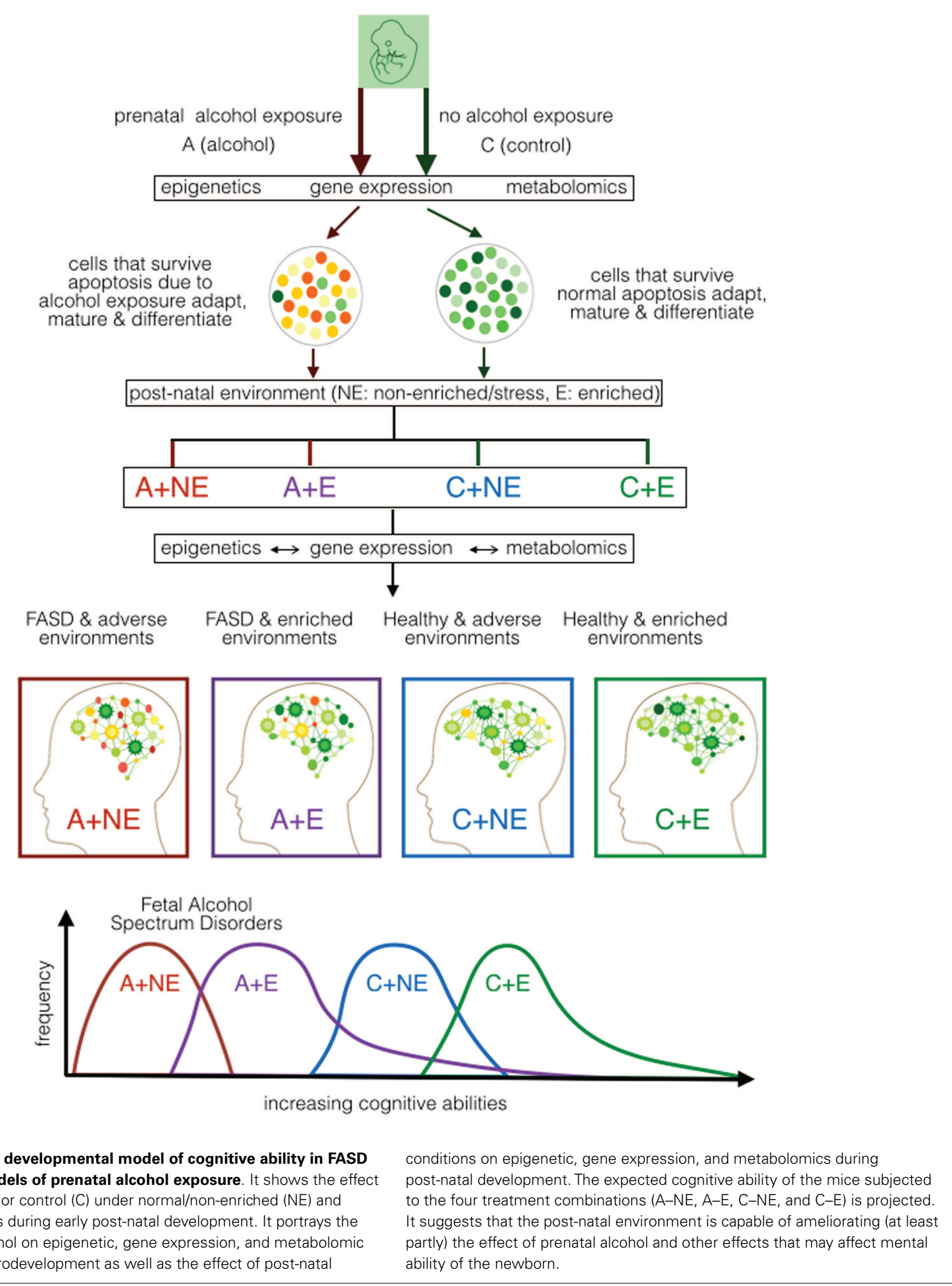

that the effectiveness of EE result from a targeted activation of specific molecular mechanisms that modify brain structure and function and are ultimately expressed as "rehabilitated" behaviors. Compared to standard housing conditions (non-enriched) with shoe-box sized cages and basic food and housing, enriched cages tend to be much larger. The latter have toys of various shapes, sizes and textures, tunnels, nesting material, heavy bedding, and access to running wheels and ladders. The objects and their locations 
are changed weekly. Such environments facilitate mice to burrow, climb, chew, run, and explore new objects and placements, thereby engaging and developing cognitive processes. To eliminate stress due to isolation and or lack of social interaction, all mice, whether in standard or enriched cages are socially housed.

Our lab, amongst others has been interested in learning more about the effects of a positive, enriched post-natal environment on mice exposed to alcohol prenatally. Our first objective has been to demonstrate that environmental enrichment can ameliorate some, if not all of the behavioral and cognitive deficits that are characteristic FASD phenotypes. Four groups of mice have been generated: Control/Saline $(\mathrm{C})$ mice living in enriched $(\mathrm{CE})$ and non-enriched (CNE) conditions and prenatal alcohol exposed mice (A) living in enriched (AE) and non-enriched (ANE) conditions. Our results show that FASD mice that have been exposed to environmental enrichment (i) exhibit a fewer number of anxiety-like traits (as evidenced by more time spent in the light-region of the light-dark box and open-arms of the elevated-plus maze) and (ii) perform relatively better in learning and memory tests (as evaluated by the novel-object recognition and the Barnes maze). This experimental design has also allowed us to establish that enrichment not only ameliorates behavioral and cognitive deficits of affected mice (AE versus ANE) but improves these characteristics in normal, healthy control mice that had never been exposed to alcohol (CE versus $\mathrm{CNE}$ ). Group comparison has also shown that prenatal ethanol exposure causes permanent and long-lasting damage to the developing brain. Further, the post-natal environmental enrichment is successful in ameliorating these deficits only to a certain extent. The mechanism involved in this amelioration is poorly understood and deserves further research.

The long-lasting effects of environmental enrichment have implicated changes in epigenetic machinery. Such results in conjunction with other lines of evidence show that the DNA methyltransferases (DNMTs) and histone acetyltransferases (HATs) are essential in neurodevelopment activities such as neural stem cell proliferation, differentiation, and synaptic plasticity (77-79). Work by Rampon et al. was among the first to show that DNMTs are preferentially up-regulated in the brains of healthy mice that have undergone environmental enrichment (80). While a number of genes involved in neuronal structure, neural plasticity, and synaptic signaling were up-regulated, the highest levels of induction was found in DNMTs. These enzymes are critical in neural cell differentiation induced by nerve growth factors (80). In 2011, Lopez-Atalaya et al. investigated the role of the histone acetyltransferase CREB-binding protein (CBP) in the context of environmental enrichment (81). CBP has been shown to be involved in neural plasticity and memory processes in the brain. Dysregulation of CBP is associated with a complex epigenetic disorder known as Rubinstein-Taybi syndrome, characterized by behavioral and cognitive deficits. CBP-deficient mice undergoing environmental enrichment have ameliorated physiological and behavioral deficits. In addition, multiple roles of CBP in neurogenesis and neuroadaptation to environmental changes were identified (81). Environmental enrichment has been shown to cause a dramatic increase in IDNA levels of BDNF, with concomitant widespread changes in histone methylation at various BDNF promoters and no change in the expression levels of several brain-specific microRNAs (82). Various other studies have pointed out the important role of BDNF in learning and memory processes $(83,84)$, particularly how BDNF shapes the cognitive and stressresponse trajectory of neurodevelopment through interactions with the HPA axis (85-88). Our lab and others are currently investigating the effects of environmental enrichment on mice following alcohol exposure in the context of BDNF and its associated epigenetic marks to gain a better understanding of how the post-natal environment acts to ameliorate negative phenotypic outcomes as a result of alcohol exposure.

\section{SYNTHESIS AND FUTURE PERSPECTIVE}

Most intellectual disabilities in children are caused by neurodevelopmental aberrations. Often they involve complex interactions of genes and environment over prenatal and post-natal periods. For example, intellectual disabilities in FASD are caused by PAE that disrupts neurodevelopment via alterations in gene expression. This affects a number of pathways that undergo changes during ontogeny over time. Here, the primary effect of alcohol covers cellular compromise and apoptosis, the expected toxic effect of ethanol. It leaves a molecular footprint that is shared among neurological disorders. The genes affected are related via hub molecules. More important, these results may last for life. We attribute them to epigenetic changes. The epigenetic machinery affected includes DNA methylation, miRNA, and histone modifications (44). The results argue that epigenetic features are critical during neurodevelopment. Any aberration in ongoing epigenetic marks at any stage during neurodevelopment may result in intellectual disabilities. It follows reports that have implicated epigenetic causes in intellectual disabilities (89-91). Such a conclusion has far reaching implications including prospect for an epigenetic therapy (92). We anticipate that this will be a major challenge for the scientific community in the next decade.

We argue that relatively long time course of neurodevelopment offers an opportunity to apply potential epigenetic therapy in intellectual disabilities. For example, a prenatal defect may be corrected following birth during early post-natal development. At this stage, developing brain is malleable. Also, it is responsive to variety of mediators including drugs, care, and social interactions. As stated, it is possible to partially ameliorate FASD deficits by post-natal environmental enrichment in B6 mice. The current most logical mediator for any amelioration in humans appears to be the early environment enrichment including cognitive therapy and interactive schooling. It is considered most logical and effective.

Rodent research also suggests that animals raised under environmentally enriched conditions exhibit relatively fewer stressand anxiety-like traits. Also, they have improved learning and memory. Further, just like the effect of prenatal alcohol, the recovery of FASD-related cognitive dysfunction due to post-natal environment also involves epigenetic processes. Such results are encouraging for the reversal of epigenetic marks. Although the specific methods for this reversal are not apparent, rehabilitative therapies and drug regimes that target epigenetic pathways would provide a good starting point. To this end, the further research should clarify two aspects of this research. First, what is the relationship between DNA methylation, histone modification and microRNA expression, brain structure and function, 
and intellectual ability including intellectual deficits in the FASD model? Second, what are the genetic pathways and mechanisms that might be targeted in future attempts to treat behavioral, cognitive, and intellectual deficits associated with human fetal alcohol exposure? The answer to such questions will have the potential to identify suitable treatments for ID caused by neurodevelopmental aberrations.

\section{ACKNOWLEDGMENTS}

We are grateful to David Carter and the London Regional Genomics Centre and to Morgan L. Kleiber, Eric J. Diehl, Benjamin I. Laufer, Katarzyna Mantha, and Randa Stringer for their contributions to this research. This research was supported by funding from grants from the Natural Sciences and Engineering Research Council of Canada (NSERC), Canadian Institutes of Health Research (CIHR), and the Ontario Mental Health Foundation (OMHF) to Shiva M. Singh.

\section{REFERENCES}

1. Papazoglou A, Jacobson LA, McCabe M, Kaufmann W, Zabel TA. To ID or not to ID? Changes in classification rates of intellectual disability using DSM-5. Intellect Dev Disabil (2014) 52(3):165-74. doi:10.1352/1934-9556-52.3.165

2. The 2006 Participation and Activity Limitation Survey: Disability in Canada (89628-X) (2010). Number 15. Available from: http://www5.statcan.gc.ca/olc-cel/ olc.action?objId=89-628-X\&objType $=2$ \&lang=en\&limit $=0$

3. Centers for Disease Control and Prevention (CDC). Economic costs associated with intellectual disability, cerebral palsy, hearing loss, and vision impairment - United States, 2003. MMWR Morb Mortal Wkly Rep (2004) 53(3):57-9. doi:10.1016/s1479-3547(03)03011-2

4. Dekker AD, De Deyn PP, Rots MG. Epigenetics: the neglected key to minimize learning and memory deficits in Down syndrome. Neurosci Biobehav Rev (2014) 45C:72-84. doi:10.1016/j.neubiorev.2014.05.004

5. Das D, Phillips C, Hsieh W, Sumanth K, Dang V, Salehi A. Neurotransmitterbased strategies for the treatment of cognitive dysfunction in Down syndrome. Prog Neuropsychopharmacol Biol Psychiatry (2014) 54C:140-8. doi:10.1016/j. pnpbp.2014.05.004

6. Maurin T, Zongaro S, Bardoni B. Fragile X syndrome: from molecular pathology to therapy. Neurosci Biobehav Rev (2014). doi:10.1016/j.neubiorev.2014.01.006

7. Blau N, MacDonald A, van Spronsen F. There is no doubt that the early identification of PKU and prompt and continuous intervention prevents intellectual disability in most patients. Mol Genet Metab (2011) 104(Suppl):S1. doi:10.1016/j.ymgme.2011.10.007

8. Nissenkorn A, Michelson M, Ben-Zeev B, Lerman-Sagie T. Inborn errors of metabolism: a cause of abnormal brain development. Neurology (2001) 56(10):1265-72. doi:10.1212/WNL.56.10.1265

9. Raymond FL, Tarpey P. The genetics of intellectual disability. Hum Mol Genet (2006) 15:R110-6. doi:10.1093/hmg/ddl189

10. Thompson BL, Levitt P, Stanwood GD. Prenatal exposure to drugs: effects on brain development and implications for policy and education. Nat Rev Neurosci (2009) 10(4):303-12. doi:10.1038/nrn2598

11. Kodituwakku PW. Defining the behavioral phenotype in children with fetal alcohol spectrum disorders: a review. Neurosci Biobehav Rev (2007) 31(2):192-201. doi:10.1016/j.neubiorev.2006.06.020

12. Abel EL, Sokol RJ. Maternal and fetal characteristics affecting alcohol's teratogenicity. Neurobehav Toxicol Teratol (1986) 8(4):329-34.

13. Popova S, Lange S, Burd L, Chudley AE, Clarren SK, Rehm J. Cost of fetal alcohol spectrum disorder diagnosis in Canada. PLoS One (2013) 8(4):e60434. doi:10.1371/journal.pone.0060434

14. Canadian Alcohol and Drug Use Monitoring Survey: Summary of Results for 2012 - Health Canada (2014). Available from: http://www.hc-sc.gc.ca/hc-ps/ drugs-drogues/stat/_2012/summary-sommaire-eng.php

15. Dow-Clarke RA, MacCalder L, Hessel PA. Health behaviors of pregnant women in Fort McMurray, Alberta. Can J Public Health (1994) 85(1):33-6.

16. Muckle G, Laflamme D, Gagnon J, Boucher O, Jacobson JL, Jacobson SW. Alcohol, smoking, and drug use among Inuit women of childbearing age during pregnancy and the risk to children. Alcohol Clin Exp Res (2011) 35(6):1081-91. doi:10.1111/j.1530-0277.2011.01441.x

17. Roberts G, Nanson JL. Best Practices: Fetal Alcohol Syndrome/Fetal Alcohol Effects and the Effects of Other Substance Use During Pregnancy. Ottawa, ON: Health Canada (2000).

18. Public Health Agency of Canada (PHAC). Fetal Alcohol Spectrum Disorder (FASD): A Framework for Action. Ottawa, ON: Public Health Agency of Canada (2003).

19. Williams RJ, Odaibo FS, McGee JM. Incidence of fetal alcohol syndrome in northeastern Manitoba. Can J Public Health (1999) 90(3):192-4.

20. Lange S, Shield K, Rehm J, Popova S. Prevalence of fetal alcohol spectrum disorders in child care settings: a meta-analysis. Pediatrics (2013) 132(4):e980-95. doi:10.1542/peds.2013-0066

21. Singh S, Sedgh G, Hussain R. Unintended pregnancy: worldwide levels, trends, and outcomes. Stud Fam Plann (2010) 41(4):241-50. doi:10.1111/j.1728-4465. 2010.00250.x

22. Ethen MK, Ramadhani TA, Scheuerle AE, Canfield MA, Wyszynski DF, Druschel $\mathrm{CM}$, et al. Alcohol consumption by women before and during pregnancy. Matern Child Health J (2009) 13(2):274-85. doi:10.1007/s10995-008-0328-2

23. Centers for Disease Control and Prevention (CDC). Fetal alcohol syndrome Alaska, Arizona, Colorado, and New York, 1995-1997. MMWR Morb Mortal Wkly Rep (2002) 51(20):433-5. doi:10.1001/jama.288.1.38-jwr0703-2-1

24. Gilliam DM, Kotch LE, Dudek BC, Riley EP. Ethanol teratogenesis in selectivity bred long-sleep and short-sleep mice: a comparison to inbred C57BL/6J mice. Alcohol Clin Exp Res (1989) 13(5):667-72.

25. Allan AM, Chynoweth J, Tyler LA, Caldwell KK. A mouse model of prenatal ethanol exposure using a voluntary drinking paradigm. Alcohol Clin Exp Res (2003) 27(12):2009-16. doi:10.1097/01.ALC.0000100940.95053.72

26. Boehm SLII, Moore EM, Walsh CD, Gross CD, Cavelli AM, Gigante E, et al. Using drinking in the dark to model prenatal binge-like exposure to ethanol in C57BL/6J mice. Dev Psychobiol (2008) 50(6):566-78. doi:10.1002/dev.20320

27. Kleiber ML, Wright E, Singh SM. Maternal voluntary drinking in C57BL/6J mice: advancing a model for fetal alcohol spectrum disorders. Behav Brain Res (2011) 223(2):376-87. doi:10.1016/j.bbr.2011.05.005

28. Kleiber ML, Laufer BI, Wright E, Diehl EJ, Singh SM. Long-term alterations to the brain transcriptome in a maternal voluntary consumption model of fetal alcohol spectrum disorders. Brain Res (2012) 1458:18-33. doi:10.1016/j.brainres.2012. 04.016

29. Mantha K, Kleiber M, Singh S. Neurodevelopmental timing of ethanol exposure may contribute to observed heterogeneity of behavioral deficits in a mouse model of fetal alcohol spectrum disorder (FASD). J Behav Brain Sci (2013) 3:85-99. doi:10.4236/jbbs.2013.31009

30. Kleiber ML, Mantha K, Stringer RL, Singh SM. Neurodevelopmental alcohol exposure elicits long-term changes to gene expression that alter distinct molecular pathways dependent on timing of exposure. J Neurodev Disord (2013) 5(1):6. doi:10.1186/1866-1955-5-6

31. Ikonomidou C, Bittigau P, Ishimaru MJ, Wozniak DF, Koch C, Genz K, et al. Ethanol-induced apoptotic neurodegeneration and fetal alcohol syndrome. Science (2000) 287(5455):1056-60. doi:10.1126/science.287.5455.1056

32. Parnell SE, Holloway HE, Baker LK, Styner MA, Sulik KK. Dysmorphogenic effects of first trimester-equivalent ethanol exposure in mice: a magnetic resonance microscopy-based study. Alcohol Clin Exp Res (2014) 38(7):2008-14. doi:10.1111/acer.12464

33. Nadel L, Hupbach A, Gomez R, Newman-Smith K. Memory formation, consolidation and transformation. Neurosci Biobehav Rev (2012) 36(7):1640-5. doi:10.1016/j.neubiorev.2012.03.001

34. Cho YH, Friedman E, Silva AJ. Ibotenate lesions of the hippocampus impair spatial learning but not contextual fear conditioning in mice. Behav Brain Res (1999) 98(1):77-87. doi:10.1016/S0166-4328(98)00054-0

35. Murawski NJ, Jablonski SA, Brown KL, Stanton ME. Effects of neonatal alcohol dose and exposure window on long delay and trace eyeblink conditioning in juvenile rats. Behav Brain Res (2013) 236(1):307-18. doi:10.1016/j.bbr.2012. 08.025

36. Ornoy A, Ergaz Z. Alcohol abuse in pregnant women: effects on the fetus and newborn, mode of action and maternal treatment. Int J Environ Res Public Health (2010) 7(2):364-79. doi:10.3390/ijerph7020364

37. Andersen AM, Andersen PK, Olsen J, Gronbaek M, Strandberg-Larsen K. Moderate alcohol intake during pregnancy and risk of fetal death. Int J Epidemiol (2012) 41(2):405-13. doi:10.1093/ije/dyr189 
38. Martinez-Frias ML, Bermejo E, Rodriguez-Pinilla E, Frias JL. Risk for congenital anomalies associated with different sporadic and daily doses of alcohol consumption during pregnancy: a case-control study. Birth Defects Res A Clin Mol Teratol (2004) 70(4):194-200. doi:10.1002/bdra.20017

39. Riar AK, Narasimhan M, Rathinam ML, Vedpathak D, Mummidi S, Henderson GI, et al. Ethanol-induced transcriptional activation of programmed cell death $4(\mathrm{Pdcd} 4)$ is mediated by GSK-3beta signaling in rat cortical neuroblasts. PLoS One (2014) 9(5):e98080. doi:10.1371/journal.pone.0098080

40. Pascual M, Valles SL, Renau-Piqueras J, Guerri C. Ceramide pathways modulate ethanol-induced cell death in astrocytes. J Neurochem (2003) 87(6):1535-45. doi:10.1046/j.1471-4159.2003.02130.x

41. Veazey KJ, Carnahan MN, Muller D, Miranda RC, Golding MC. Alcoholinduced epigenetic alterations to developmentally crucial genes regulating neural stemness and differentiation. Alcohol Clin Exp Res (2013) 37(7):1111-22. doi:10.1111/acer.12080

42. Kleiber ML, Diehl EJ, Laufer BI, Mantha K, Chokroborty-Hoque A, Alberry B, et al. Long-term genomic and epigenomic dysregulation as a consequence of prenatal alcohol exposure: a model for fetal alcohol spectrum disorders. Front Genet (2014) 5:161. doi:10.3389/fgene.2014.00161

43. Mantha K, Laufer BI, Singh SM. Molecular changes during neurodevelopment following second-trimester binge ethanol exposure in a mouse model of fetal alcohol spectrum disorder: from immediate effects to long-term adaptation. Dev Neurosci (2014) 36(1):29-43. doi:10.1159/000357496

44. Laufer BI, Mantha K, Kleiber ML, Diehl EJ, Addison SM, Singh SM. Long-lasting alterations to DNA methylation and ncRNAs could underlie the effects of fetal alcohol exposure in mice. Dis Model Mech (2013) 6(4):977-92. doi:10.1242/ dmm.010975

45. McEwen BS. Protection and damage from acute and chronic stress: allostasis and allostatic overload and relevance to the pathophysiology of psychiatric disorders. Ann N Y Acad Sci (2004) 1032:1-7. doi:10.1196/annals.1314.001

46. Meaney MJ, Aitken DH, Viau V, Sharma S, Sarrieau A. Neonatal handling alters adrenocortical negative feedback sensitivity and hippocampal type II glucocorticoid receptor binding in the rat. Neuroendocrinology (1989) 50(5):597-604.

47. Viau V, Sharma S, Plotsky PM, Meaney MJ. Increased plasma ACTH responses to stress in nonhandled compared with handled rats require basal levels of corticosterone and are associated with increased levels of ACTH secretagogues in the median eminence. J Neurosci (1993) 13(3):1097-105.

48. Bhatnagar S, Mitchell JB, Betito K, Boksa P, Meaney MJ. Effects of chronic intermittent cold stress on pituitary adrenocortical and sympathetic adrenomedullary functioning. Physiol Behav (1995) 57(4):633-9. doi:10.1016/ 0031-9384(94)00161-8

49. Meaney MJ, Aitken DH, van Berkel C, Bhatnagar S, Sapolsky RM. Effect of neonatal handling on age-related impairments associated with the hippocampus. Science (1988) 239(4841 Pt 1):766-8.

50. Meaney MJ, Aitken DH, Sharma S, Viau V. Basal ACTH, corticosterone and corticosterone-binding globulin levels over the diurnal cycle, and age-related changes in hippocampal type I and type II corticosteroid receptor binding capacity in young and aged, handled and nonhandled rats. Neuroendocrinology (1992) 55(2):204-13. doi:10.1159/000126116

51. Sanchez MM, Noble PM, Lyon CK, Plotsky PM, Davis M, Nemeroff CB, et al. Alterations in diurnal cortisol rhythm and acoustic startle response in nonhuman primates with adverse rearing. Biol Psychiatry (2005) 57(4):373-81. doi:10.1016/j.biopsych.2004.11.032

52. Heim C, Newport DJ, Wagner D, Wilcox MM, Miller AH, Nemeroff CB. The role of early adverse experience and adulthood stress in the prediction of neuroendocrine stress reactivity in women: a multiple regression analysis. Depress Anxiety (2002) 15(3):117-25. doi:10.1002/da.10015

53. Rao U, Hammen C, Ortiz LR, Chen LA, Poland RE. Effects of early and recent adverse experiences on adrenal response to psychosocial stress in depressed adolescents. Biol Psychiatry (2008) 64(6):521-6. doi:10.1016/j.biopsych.2008.05.012

54. Liu D, Diorio J, Day JC, Francis DD, Meaney MJ. Maternal care, hippocampal synaptogenesis and cognitive development in rats. Nat Neurosci (2000) 3(8):799-806. doi:10.1038/77702

55. Weaver IC, Cervoni N, Champagne FA, D’Alessio AC, Sharma S, Seckl JR, et al. Epigenetic programming by maternal behavior. Nat Neurosci (2004) 7(8):847-54. doi:10.1038/nn1276

56. Meaney MJ, Szyf M, Seckl JR. Epigenetic mechanisms of perinatal programming of hypothalamic-pituitary-adrenal function and health. Trends Mol Med (2007) 13(7):269-77. doi:10.1016/j.molmed.2007.05.003
57. McGowan PO, Sasaki A, D’Alessio AC, Dymov S, Labonte B, Szyf M, et al. Epigenetic regulation of the glucocorticoid receptor in human brain associates with childhood abuse. Nat Neurosci (2009) 12(3):342-8. doi:10.1038/nn. 2270

58. Meaney MJ, Szyf M. Environmental programming of stress responses through DNA methylation: life at the interface between a dynamic environment and a fixed genome. Dialogues Clin Neurosci (2005) 7(2):103-23. doi:10.1002/da. 10015

59. Warren KR, Foudin LL. Alcohol-related birth defects - the past, present, and future. Alcohol Res Health (2001) 25(3):153-8. doi:10.1037/e603882012-001

60. Roberts AD, Moore CF, DeJesus OT, Barnhart TE, Larson JA, Mukherjee J, et al. Prenatal stress, moderate fetal alcohol, and dopamine system function in rhesus monkeys. Neurotoxicol Teratol (2004) 26(2):169-78. doi:10.1016/j.ntt. 2003.12.003

61. Schneider ML, Moore CF, Gajewski LL, Larson JA, Roberts AD, Converse AK, et al. Sensory processing disorder in a primate model: evidence from a longitudinal study of prenatal alcohol and prenatal stress effects. Child Dev (2008) 79(1):100-13. doi:10.1111/j.1467-8624.2007.01113.x

62. Hellemans KG, Sliwowska JH, Verma P, Weinberg J. Prenatal alcohol exposure: fetal programming and later life vulnerability to stress, depression and anxiety disorders. Neurosci Biobehav Rev (2010) 34(6):791-807. doi:10.1016/j. neubiorev.2009.06.004

63. Haley DW, Handmaker NS, Lowe J. Infant stress reactivity and prenatal alcohol exposure. Alcohol Clin Exp Res (2006) 30(12):2055-64. doi:10.1111/j.15300277.2006.00251.x

64. Schneider ML, Moore CF, Kraemer GW, Roberts AD, DeJesus OT. The impact of prenatal stress, fetal alcohol exposure, or both on development: perspectives from a primate model. Psychoneuroendocrinology (2002) 27(1-2):285-98. doi:10.1016/S0306-4530(01)00050-6

65. Weinberg J, Sliwowska JH, Lan N, Hellemans KG. Prenatal alcohol exposure: foetal programming, the hypothalamic-pituitary-adrenal axis and sex differences in outcome. J Neuroendocrinol (2008) 20(4):470-88. doi:10.1111/j.13652826.2008.01669.x

66. Hellemans KG, Verma P, Yoon E, Yu W, Weinberg J. Prenatal alcohol exposure increases vulnerability to stress and anxiety-like disorders in adulthood. Ann N Y Acad Sci (2008) 1144:154-75. doi:10.1196/annals.1418.016

67. Franklin TB, Russig H, Weiss IC, Graff J, Linder N, Michalon A, et al. Epigenetic transmission of the impact of early stress across generations. Biol Psychiatry (2010) 68(5):408-15. doi:10.1016/j.biopsych.2010.05.036

68. Romeo RD, Mueller A, Sisti HM, Ogawa S, McEwen BS, Brake WG. Anxiety and fear behaviors in adult male and female C57BL/6 mice are modulated by maternal separation. Horm Behav (2003) 43(5):561-7. doi:10.1016/S0018-506X(03) 00063-1

69. Popova NK, Morozova MV, Naumenko VS. Ameliorative effect of BDNF on prenatal ethanol and stress exposure-induced behavioral disorders. Neurosci Lett (2011) 505(2):82-6. doi:10.1016/j.neulet.2011.09.066

70. Gross CM, Flubacher A, Tinnes S, Heyer A, Scheller M, Herpfer I, et al. Early life stress stimulates hippocampal reelin gene expression in a sex-specific manner: evidence for corticosterone-mediated action. Hippocampus (2012) 22(3):409-20. doi:10.1002/hipo.20907

71. Fenoglio KA, Brunson KL, Baram TZ. Hippocampal neuroplasticity induced by early-life stress: functional and molecular aspects. Front Neuroendocrinol (2006) 27(2):180-92. doi:10.1016/j.yfrne.2006.02.001

72. Streissguth AP, Barr HM, Sampson PD, Bookstein FL. Prenatal alcohol and offspring development: the first fourteen years. Drug Alcohol Depend (1994) 36(2):89-99.

73. Streissguth AP, O’Malley K. Neuropsychiatric implications and long-term consequences of fetal alcohol spectrum disorders. Semin Clin Neuropsychiatry (2000) 5(3):177-90. doi:10.1053/scnp.2000.6729

74. Kodituwakku PW. A neurodevelopmental framework for the development of interventions for children with fetal alcohol spectrum disorders. Alcohol (2010) 44(7-8):717-28. doi:10.1016/j.alcohol.2009.10.009

75. Peadon E, Rhys-Jones B, Bower C, Elliott EJ. Systematic review of interventions for children with fetal alcohol spectrum disorders. BMC Pediatr (2009) 9:35. doi:10.1186/1471-2431-9-35

76. van Praag H, Kempermann G, Gage FH. Neural consequences of environmental enrichment. Nat Rev Neurosci (2000) 1(3):191-8. doi:10.1038/35042057

77. Sweatt JD. The emerging field of neuroepigenetics. Neuron (2013) 80(3):624-32. doi:10.1016/j.neuron.2013.10.023 
78. Zovkic IB, Guzman-Karlsson MC, Sweatt JD. Epigenetic regulation of memory formation and maintenance. Learn Mem (2013) 20(2):61-74. doi:10.1101/lm. 026575.112

79. Day JJ, Sweatt JD. Epigenetic mechanisms in cognition. Neuron (2011) 70(5):813-29. doi:10.1016/j.neuron.2011.05.019

80. Rampon C, Jiang CH, Dong H, Tang YP, Lockhart DJ, Schultz PG, et al. Effects of environmental enrichment on gene expression in the brain. Proc Natl Acad Sci U S A (2000) 97(23):12880-4. doi:10.1073/pnas.97.23.12880

81. Lopez-Atalaya JP, Gervasini C, Mottadelli F, Spena S, Piccione M, Scarano G, et al. Histone acetylation deficits in lymphoblastoid cell lines from patients with Rubinstein-Taybi syndrome. J Med Genet (2012) 49(1):66-74. doi:10.1136/ jmedgenet-2011-100354

82. Kuzumaki N, Ikegami D, Tamura R, Hareyama N, Imai S, Narita M, et al. Hippocampal epigenetic modification at the brain-derived neurotrophic factor gene induced by an enriched environment. Hippocampus (2011) 21(2):127-32. doi:10.1002/hipo. 20775

83. Carlino D, De Vanna M, Tongiorgi E. Is altered BDNF biosynthesis a general feature in patients with cognitive dysfunctions? Neuroscientist (2013) 19(4):345-53. doi:10.1177/1073858412469444

84. Ernfors P, Kucera J, Lee KF, Loring J, Jaenisch R. Studies on the physiological role of brain-derived neurotrophic factor and neurotrophin-3 in knockout mice. Int J Dev Biol (1995) 39(5):799-807.

85. Finsterwald C, Alberini CM. Stress and glucocorticoid receptor-dependent mechanisms in long-term memory: from adaptive responses to psychopathologies. Neurobiol Learn Mem (2014) 112C:17-29. doi:10.1016/j.nlm.2013. 09.017

86. Kunugi H, Hori H, Adachi N, Numakawa T. Interface between hypothalamicpituitary-adrenal axis and brain-derived neurotrophic factor in depression. Psychiatry Clin Neurosci (2010) 64(5):447-59. doi:10.1111/j.1440-1819.2010. 02135.x

87. Martinez-Levy GA, Cruz-Fuentes CS. Genetic and epigenetic regulation of the brain-derived neurotrophic factor in the central nervous system. Yale J Biol Med (2014) 87(2):173-86. doi:10.1016/S0306-4522(97)00315-1
88. Boulle F, van den Hove DL, Jakob SB, Rutten BP, Hamon M, van Os J, et al. Epigenetic regulation of the BDNF gene: implications for psychiatric disorders. Mol Psychiatry (2012) 17(6):584-96. doi:10.1038/mp.2011.107

89. Gibbons RJ, McDowell TL, Raman S, O’Rourke DM, Garrick D, Ayyub H, et al. Mutations in ATRX, encoding a SWI/SNF-like protein, cause diverse changes in the pattern of DNA methylation. Nat Genet (2000) 24(4):368-71. doi: $10.1038 / 74191$

90. Szyf M. The early life social environment and DNA methylation: DNA methylation mediating the long-term impact of social environments early in life. Epigenetics (2011) 6(8):971-8. doi:10.4161/epi.6.8.16793

91. Franklin TB, Mansuy IM. The involvement of epigenetic defects in intellectual disability. Neurobiol Learn Mem (2011) 96(1):61-7. doi:10.1016/j.nlm.2011. 04.001

92. Egger G, Liang G, Aparicio A, Jones PA. Epigenetics in human disease and prospects for epigenetic therapy. Nature (2004) 429(6990):457-63. doi:10.1038/ nature 02625

Conflict of Interest Statement: The authors declare that the research was conducted in the absence of any commercial or financial relationships that could be construed as a potential conflict of interest.

Received: 18 July 2014; accepted: 11 August 2014; published online: 26 August 2014. Citation: Chokroborty-Hoque A, Alberry B and Singh SM (2014) Exploring the complexity of intellectual disability in fetal alcohol spectrum disorders. Front. Pediatr. 2:90. doi: $10.3389 /$ fped.2014.00090

This article was submitted to Child and Neurodevelopmental Psychiatry, a section of the journal Frontiers in Pediatrics.

Copyright (c) 2014 Chokroborty-Hoque, Alberry and Singh. This is an open-access article distributed under the terms of the Creative Commons Attribution License (CC BY). The use, distribution or reproduction in other forums is permitted, provided the original author(s) or licensor are credited and that the original publication in this journal is cited, in accordance with accepted academic practice. No use, distribution or reproduction is permitted which does not comply with these terms. 